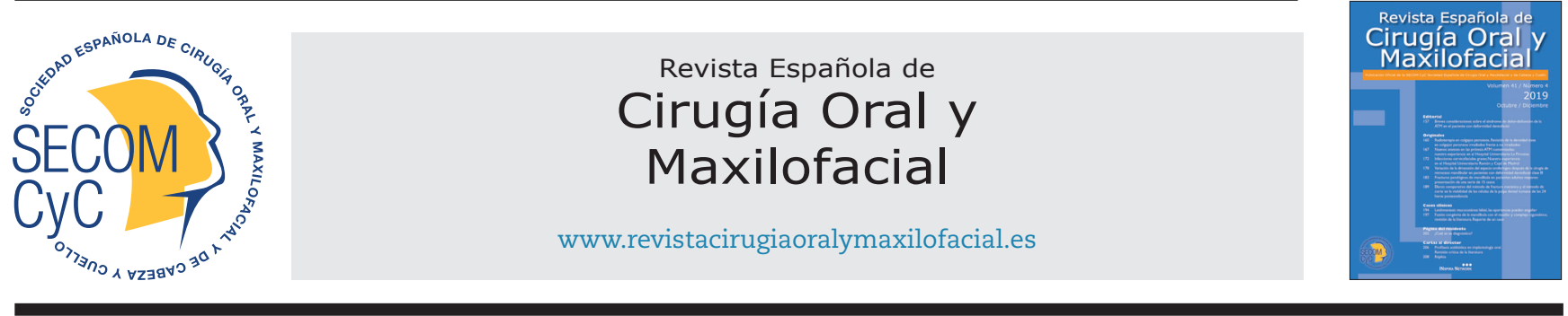

\title{
Original
}

\section{Efecto comparativo del método de fractura mecánica y el método de corte en la viabilidad de las células de la pulpa dental humana de las 24 horas postexodoncia}

\author{
Xiomara Flores-Cóndor y Esperanza R. Ayón-Haro* \\ Laboratorio de Investigación en Biología Oral y Molecular, Facultad de Odontología de la Universidad de San Martín de Porres, Lima, \\ Perú
}

\section{INFORMACIÓN DEL ARTÍCULO}

Historia del artículo:

Recibido el 3 de octubre de 2017

Aceptado el 17 de marzo de 2018

Palabras clave:

Pulpa dental, viabilidad celular, recuento de células.

\section{R E S U M E N}

Objetivo: En los estudios sobre biomateriales e ingeniería de tejidos se emplean células de la pulpa dental para regenerar o sustituir las deficiencias de tejido óseo en la cavidad oral. Para evaluar su potencial clínico se utilizan modelos de cultivo celular, en los cuales se aplican diversos métodos de extracción de la pulpa dental. Pero aún no está claro qué método es el más conveniente. Por ello, el propósito de este estudio fue comparar el método de fractura mecánica y el método de corte en la viabilidad celular.

Material y métodos: Se extrajo el tejido pulpar de dientes completamente desarrollados y sanos de 32 pacientes de entre 18 y 40 años mediante dos técnicas: el método de fractura mecánica (grupo 1) y el método de corte (grupo 2). Para determinar la viabilidad celular se usó el colorante azul de tripán. Las células teñidas de azul (muertas) y las células birrefringentes (vivas) fueron contabilizadas en el microscopio óptico.

Resultados: Al emplear el método de fractura mecánica se obtuvo un 87,72 \% de viabilidad celular, mientras que al aplicar el método de corte se logró una menor viabilidad: 83,59 \%. $\mathrm{Al}$ analizar los datos ( $\mathrm{n}=16$ por grupo) bajo la prueba $\mathrm{t}$ de Student se obtuvo una diferencia significativa $(p=0,006)$.

Conclusiones: El método de fractura mecánica permite obtener una viabilidad celular más alta en comparación al método de corte, empleado en dientes extraídos dentro de las 24 horas.

${ }^{*}$ Corresponding author:

E-mail address: eayonh@usmp.pe (Esperanza R. Ayón-Haro).

DOI: 10.20986/recom.2019.1023/2019

1130-0558/@ 2019 SECOM. Publicado por Inspira Network. Este es un artículo Open Access bajo la licencia CC BY-NC-ND (http:// creativecommons.org/licenses/by-nc-nd/4.0/). 


\section{Comparative effect of the mechanical fracture method and the cutting method on the viability of human dental pulp cells from teeth extracted within 24 hours}

Keywords:

Dental pulp, cell survival, cell count.

\section{A B S T R A C T}

Aim: In biomaterials and tissue engineering studies, dental pulp cells are used to regenerate or replace deficiencies of bone tissue in the oral cavity. To evaluate its clinical potential, cell culture models are employed, in which different extraction methods of the dental pulp are applied. Mainly, the tooth is broken by fracture mechanics and the cutting method to obtain the dental pulp. However, it is not clear yet which method is the most convenient. Therefore, the purpose of this study was to compare the effect of two methods of fracture mechanical and cutting methods on the dental pulp cell viability.

Methodology: Dental pulp tissue was removed of fully developed and healthy teeth of 32 patients between 18 to 40 years, through two techniques: the methods of fracture mechanics (group 1) and cutting (group 2). To determine the cell viability, trypan blue dye was used. The cells were counted in the microscope, blue stained cells (dead) and birefringent (living). Results: Using the mechanical fracture method, $87.72 \%$ of cell viability was obtained. While with the cutting method was achieved a lower viability $83.59 \%$. There was a significant difference $(p=0.006)$ when analyzing the data $(n=16$ per group) under $t$-Student test.

Conclusions: The use of mechanical fracture method for the extraction of the dental pulp tissue allows to obtain higher percentages of cell viability compared to cutting method.

\section{INTRODUCCIÓN}

En la última década las investigaciones en odontología se han concentrado en el desarrollo de nuevos materiales biológicos a partir de la introducción de métodos de cultivo celular y cultivo de tejidos ${ }^{1}$. La pulpa dental se compone de tejido conectivo vascular con fibroblastos, odontoblastos, macrófagos, células dendríticas, linfocitos y una población de células madre que ayudan en la reparación de la dentina circundante². Este tejido es una de las principales fuentes para la obtención de células, ya que tiene la ventaja de presentar un fácil acceso debido a que se consigue después de una extracción dentaria con fines ortodónticos o como procedimiento de impactación ${ }^{3}$. Por ello se han realizado estudios de viabilidad en fibroblastos y células madre de la pulpa dental para evaluar la citotoxicidad de materiales dentales, tales como resinas compuestas, cementos de endodoncia y productos odontológicos a base de plantas ${ }^{4-8}$. En los últimos años los productos basados en células mesenquimales han sido desarrollados para mejorar las terapias quirúrgicas en numerosas disciplinas médicas, ofreciendo nuevas modalidades de tratamiento con células madre de la pulpa dental, que incluyen procedimientos de aumento óseo en la cirugía oral para la colocación de implantes dentales y para reconstrucciones periodontales, entre otros ${ }^{9,10}$. En estudios previos de aislamiento y cultivo celular de la pulpa dental se han empleado varios métodos para obtener el tejido pulpar después de la extracción dental, como son: el método de fractura mecánica con martillo² ${ }^{2}$ el método de corte con baja velocidad ${ }^{11,12}$, el método de fractura con fórceps ${ }^{13-15}$ y el de extracción de la pulpa a través del ápice mediante limas ${ }^{16}$. Sin embargo, aún no se ha llegado a un consenso que establezca qué método de extracción pulpar es el más adecuado en cuanto a la viabilidad celular.
Los estudios de viabilidad celular constituyen un importante control de calidad, especialmente en aquellas poblaciones celulares con un alto potencial para su utilización en ingeniería tisular. Existen varias técnicas para evaluar la viabilidad celular. Los métodos basados en la permeabilidad de la membrana son los más numerosos y utilizados. El principio básico consiste en la utilización de un colorante que penetra y colorea las células vivas o muertas. El azul de tripán es un colorante orgánico usado para determinar el número de células viables presentes en una suspensión celular. Este método está basado en que las células vivas poseen una membrana celular intacta que no dejará penetrar el colorante, por lo tanto, mantendrán un citoplasma claro; a diferencia de las células muertas, que permitirán el paso del colorante y mostrarán un citoplasma azul al microscopio óptico ${ }^{17,18}$.

El primer paso para el uso de células de la pulpa dental es seleccionar el método más indicado para su obtención. Los métodos más comunes de extracción pulpar son: la fractura mecánica y el método de corte. Por consiguiente, el objetivo de esta investigación fue determinar qué método de extracción de la pulpa dental es más eficiente, el método de corte o el método de fractura mecánica, en la viabilidad celular de la pulpa dental.

\section{MATERIAL Y MÉTODOS}

Se obtuvieron 32 dientes de pacientes adultos cuyo rango de edad comprendió de 18 a 40 años, atendidos en el Centro Quirúrgico de la Clínica Especializada en Odontología de la Universidad de San Martín de Porres. Todos los pacientes fueron informados de las características del estudio y aceptaron libremente colaborar con el mismo, aportando el diente extraído, el cual fue extraído por indicación profesional. Este 
procedimiento se llevó a cabo de conformidad con las directrices dictadas por el Comité de Ética de Investigación de la Facultad de Odontología de la Universidad de San Martín de Porres. Los criterios de inclusión fueron los siguientes: dientes sanos de pacientes de entre 18 y 40 años de edad. Se excluyeron los dientes de pacientes con enfermedad sistémica no controlada, los dientes fracturados durante la exodoncia, los dientes con odontosección y los dientes con ápice abierto ${ }^{19}$. Se escogieron dientes con ápice cerrado para evitar la contaminación bacteriana del tejido pulpar expuesto al exterior y para uniformizar la condición de las muestras, y también para evitar el riesgo de obtener otro tejido (papila apical o ligamento periodontal), debido a que en este estudio se buscó utilizar únicamente la pulpa dental de dientes permanentes. Se utilizaron 32 tejidos pulpares, divididos en dos grupos de 16 dientes cada uno: método de fractura mecánica (grupo 1) y método de corte (grupo 2). Se lavó el diente extraído con minimum essential medium eagle (MEM) (Sigma-Aldrich, St. Louis, MO, USA), suplementado con penicilina-estreptomicina (Gibco, New York, NY, USA) por 20 minutos. La exposición del tejido pulpar del grupo 1 se obtuvo con un golpe de un martillo, cubriendo previamente el diente con gasa humedecida con alcohol al 70 \%. Para el grupo 2 se realizó un corte con un disco diamantado dentado montado en un micromotor. Se cortó el diente entre la línea amelocementaria y la furca a $28.000 \mathrm{rpm}$, irrigando intermitentemente con suero fisiológico. En la cabina flujolaminar se extrajo el tejido pulpar dental. La suspensión celular se centrifugó a 1.100 rpm por $10 \mathrm{minu}-$ tos. Luego se resuspendió el botón celular con MEM. Para determinar la viabilidad celular se mezclaron $10 \mu \mathrm{l}$ de la suspensión celular con $10 \mu \mathrm{l}$ de azul de tripán (Gibco-Invitrogen, Carlsbad, CA, EE.UU). Luego se procedió al recuento de las células viables y muertas en un hemocitómetro utilizando un microscopio óptico.

$\mathrm{n}^{\circ}$ de células viables $\times$ factor de dilución $\times 10.000 \times$ volumen $\mathrm{N}^{\circ}$ de células $=\frac{\mathrm{n}^{\circ} \text { de células viables } \times \text { factor de dilución } \times 10.000 \times \text { volumen }}{\mathrm{n}^{\circ} \text { de cuadrantes contados }}$

$\mathrm{n}^{\circ}$ de células viables $\times 100$

$\mathrm{n}^{\circ}$ total de células

\section{RESULTADOS}

Los resultados mostraron que al emplear el método de fractura mecánica se obtiene significativamente un mayor porcentaje de viabilidad celular en comparación al método de corte. El grupo del método de fractura mecánica presentó un 87,7 \% de viabilidad celular en promedio, con relación al grupo del método de corte, que presentó una viabilidad celular del 83,5\% en promedio. Al comparar ambos grupos se obtuvo el valor de $p=0,006$ mediante la prueba t de Student, con un nivel de confianza del $95 \%$ (Figura 1).

El número total de células obtenidas según el método de corte fue 5,74 × 107 y con el método de fractura mecánica fue 7,54 × 104 con el método de fractura mecánica; sin embargo, en el análisis estadístico no se encontró diferencia significativa $(p=0,144)$.

Para verificar si el tiempo transcurrido después de la exodoncia influye en la viabilidad celular y el número de células obtenidas de la pulpa dental se realizó la prueba Rho de Spearman.

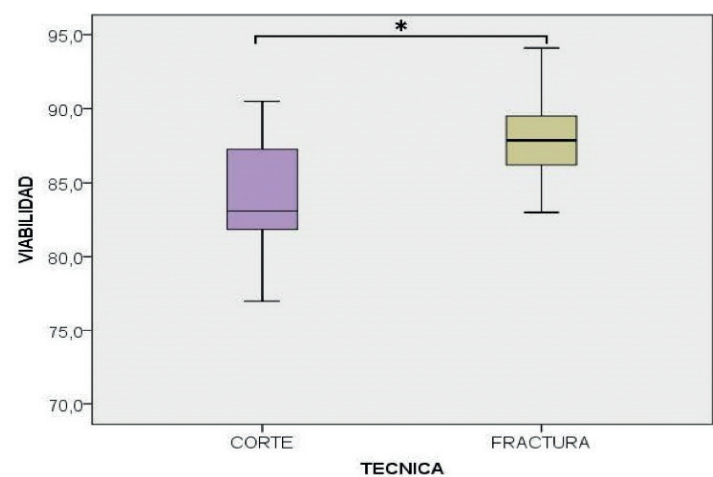

Figura 1. Comparación del porcentaje de viabilidad celular obtenida entre el método de corte y el método de fractura mecánica. Los datos son promedios \pm desviación estándar. *Existe diferencia estadísticamente significativa con $\mathrm{p}<0,05$ utilizando la prueba t de Student.

No se encontró correlación entre el tiempo de 3 a 20 horas después de la exodoncia con la viabilidad celular $(p=0,785)$ y el número de células $(p=0,321)$. De forma similar, no se encontró correlación entre la edad (de 18 a 40 años) con la viabilidad celular $(p=0,532)$ y el número de células $(p=0,944)$.

\section{DISCUSIÓN}

Según nuestros resultados, el método de fractura con martillo mostró un mayor porcentaje de viabilidad celular $(87,72 \%)$ en contraste con el método de corte (83,59\%). Al aplicar la prueba t de Student se obtuvo una diferencia significativa, lo que prueba la superioridad del método de corte para obtener células vivas. Este resultado se puede deber a que el método de fractura mecánica con martillo no provoca calor constante, lo que asegura una tasa de viabilidad celular más alta. En contraste, el método de corte produce un sobrecalentamiento del tejido, a pesar de la irrigación intermitente. Como consecuencia, muchas células de la pulpa dental mueren. Los resultados obtenidos según el método de corte difieren con lo reportado por Suchánek y cols., quienes se enfocaron en obtener células madre de la pulpa dental mediante el método de corte con una fresa diamantada de alta velocidad e irrigación constante. Ellos no lograron obtener células madre de la pulpa dental ${ }^{16}$. Sin embargo, en nuestro trabajo se tomó la precaución de usar un micromotor de baja velocidad dentro de una cabina flujolaminar, logrando obtener células de la pulpa dental, entre las que pueden encontrarse células madre de la pulpa dental. No obstante, en este estudio no se utilizó la técnica de reacción en cadena de la polimerasa y de citometría de flujo para identificar esta población celular.

En esta investigación, el número total de células obtenidas

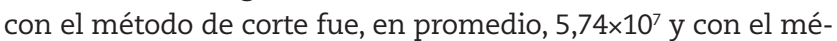
todo de fractura mecánica $7,54 \times 10^{7}$. Al aplicar la prueba t de Student no se encontraron diferencias significativas entre el número total de células de ambos métodos. No pudimos contrastar nuestros resultados con los de otros investigadores, debido a que no encontramos estudios que reportasen la 
concentración total de células de la pulpa dental. Como referencia podemos citar estudios que han trabajado solo con un tipo celular. Souza y cols. ${ }^{20}$ y Karamzadeh y cols. ${ }^{12}$ reportaron la obtención de $5 \times 10^{4}$ y $2,5 \times 10^{5}$ células madre de la pulpa dental respectivamente, utilizando el método de corte con baja velocidad. Por lo tanto, este trabajo es el primer estudio que publica un recuento de toda la población celular de la pulpa dental humana.

En nuestro estudio, el tiempo que tardamos en empezar a extraer la pulpa dental en el laboratorio después de la exodoncia fue un intervalo de 3 a 20 horas. Según la prueba Rho de Spearman no se encontró correlación entre el tiempo transcurrido desde la exodoncia con la viabilidad celular y el número total de células. Este resultado se puede atribuir a que el intervalo de tiempo evaluado fue corto (no más de 20 horas), a diferencia de Perry y cols., quienes trabajaron con una subpoblación celular específica e intervalos más grandes (de 0 a 120 horas). De acuerdo a sus resultados, se logró aislar células madre de la pulpa dental hasta 120 horas después de la exodoncia, pero se observó un declive marcado en la concentración celular a las 72 horas; de allí en adelante el número de células obtenidas fue mucho menor ${ }^{21}$.

En este trabajo se ha utilizado la pulpa dental de pacientes de entre 18 y 40 años. Al aplicar la prueba Rho de Spearman no se encontró correlación entre la edad con la viabilidad celular y el número total de células. Esto se debe probablemente a que en nuestra investigación el rango de edad fue corto. Según la bibliografía, se han obtenido células de la pulpa dental de pacientes con diferentes rangos de edad. Horibe y cols. compararon la obtención de células madre de la pulpa dental de pacientes de entre 19 y 30 años con otros pacientes de entre 44 y 70 años. El grupo de pacientes de mayor edad mostró un pequeño declive en las propiedades de las células madre: migración, potencial de diferenciación y proliferación ${ }^{22}$. Sin embargo, Bressan y cols., en su estudio con pacientes de 16 a 66 años, observaron una mejor habilidad proliferativa de las células madre de la pulpa dental en los pacientes de 25 años a más ${ }^{23}$. Considerando los estudios anteriores y los resultados de este trabajo, concluimos que la pulpa dental obtenida de pacientes jóvenes y adultos puede emplearse satisfactoriamente en los estudios basados en células.

Según Chamieh y cols., la ingeniería tisular puede proporcionar una terapia efectiva para la regeneración ósea craneofacial. Ellos demostraron que las células madre de la pulpa dental en combinación con un andamio de gel de colágeno denso beneficiaba el proceso de reparación ósea craneofacial en ratas ${ }^{10}$.

\section{CONCLUSIONES}

Según nuestro conocimiento, este es el primer trabajo que compara la viabilidad celular y el número total de células de la pulpa dental según el método de corte y el método de fractura mecánica con martillo. Nuestros resultados indican que se obtiene un porcentaje mayor de viabilidad celular con el método de fractura en comparación con el método de corte. Este trabajo contribuye a la optimización del protocolo de aislamiento y cultivo de células de la pulpa dental, el cual será útil para desarrollar pesquisas en el campo de la medicina regenerativa, ámbito que nos conduce al desarrollo de nuevas estrategias terapéuticas que regeneren tejidos orales perdidos como resultado de una enfermedad o traumatismo sufrido en el macizo craneofacial.

\section{B I B L I O G R A F Í A}

1. Zorzeto A. Avaliação in vitro do cultivo de fibroblastos gengivais humanos en matriz dérmica acelular. [Tesis de maestría]. Sao Paulo: Universidade de Sao Paulo; 2008.

2. Syed-Picard F, Ray H, Kumta P, Sfeir C. Scaffoldless tissue-engineered dental pulp cell constructs for endodontic therapy. J Dent Res. 2014;93(3):250-5. DOI: 10.1177/0022034513517901.

3. Avinash K, Malaippan S, Dooraiswamy JN. Methods of isolation and characterization of stem cells from different regions of oral cavity using markers: a systematic review. Int J Stem Cells. 2017;10(1):12-20. DOI: 10.15283/ijsc17010.

4. Zare M, Ranjbarian P, Shiravi S. Cytotoxicity evaluation of iranian propolis and calcium hydroxide on dental pulp fibroblasts. J Dent Res. 2014;8(3):130-3. DOI: 10.5681/joddd.2014.024.

5. Shafiei F, Tavangar MS, Razmkhah M, Attar A, Alavi A. Cytotoxic effect of silorane and methacrylate based composites on the human dental pulp stem cells and fibroblasts. Med Oral Patol Oral Cir Bucal. 2014;19(4):350-8. DOI: 10.4317/medoral.19340.

6. Rozas C. Evaluación de la citotoxicidad de dos cementos ionómeros vítreos en un modelo experimental de fibroblastos gingivales humanos. Estudio estructural y microanalítico. [Tesis doctoral]. Córdoba: Universidad de Córdoba; 2010.

7. Quezada R. Citotoxicidad del aloe vera y el formocresol en las células mesenquimales de pulpa dental. [Tesis de maestría]. Nuevo León: Universidad Autónoma de Nuevo León; 2014.

8. Tetè S, Zizzari VL, Borelli B, De Colli M, Zara S, Sorrentino R, et al. Proliferation and adhesion capability of human gingival fibroblasts onto zirconia, lithium disilicate and feldspathic veneering ceramic in vitro. Dent Mater J. 2014;33(1):7-15. DOI: 10.4012/dmj.2013-185.

9. Ducret M, Fabre H, Degoul O, Atzeni G, McGuckin C, Forraz N, et al. Manufacturing of dental pulp cell-based products from human third molars: current strategies and future investigations. Front Physiol. 2015;6:213. DOI: 10.3389/fphys.2015.00213.

10. Chamieh F, Collignon AM, Coyac BR, Lesieur J, Ribes S, Sadoine $J$, et al. Accelerated craniofacial bone regeneration through dense collagen gel scaffolds seeded with dental pulp stem cells. Sci Rep. 2016;6:38814. DOI: 10.1038/srep38814.

11. Woods EJ, Perry BC, Hockema JJ, Larson L, Zhou D, Goebel WS. Optimized cryopreservation method for human dental pulpderived stem cells and their tissues of origin for banking and clinical use. Cryobiology. 2009;59(2):150-7. DOI: 10.1016/j.cryobiol.2009.06.005.

12. Karamzadeh R, Eslaminejad MB, Aflatoonian R. Isolation, characterization and comparative differentiation of human dental pulp stem cells derived from permanent teeth by using two different methods. J Vis Exp. 2012;(69):43-72. DOI: 10.3791/4372.

13. Pierdomenico L, Bonsi L, Calvitti M, Rondelli D, Arpinati M, Chirumbolo G, et al. Multipotent mesenchymal stem cells with immunosuppressive activity can be easily isolated from dental pulp. Transplantation. 2005;80(6):836-42. DOI; 10.1097/01. tp.0000173794.72151.88.

14. Hilkens P, Fanton Y, Martens W, Gervois P, Struys T, Politis C, et al. Pro-angiogenic impact of dental stem cells in vitro and in vivo. Stem Cell Res. 2014;12(3):778-90. DOI: 10.1016/j. scr.2014.03.008.

15. Agha-Hosseini F, Jahani M, Jahani M, Mirzaii-Dizgah I, AliMoghaddam K. In vitro isolation of stem cells derived from human dental pulp. Clin Transplant. 2010;24(2):23-8. DOI: 10.1111/j.1399-0012.2009.01137.x. 
16. Suchánek J, Soukup T, Ivancaková R, Karbanová J, Hubková V, Pytlík R, et al. Human dental pulp stem cells - Isolation and long term cultivation. Acta Medica (Hradec Kralove). 2007;50(3):195-201.

17. Strober W. Trypan blue exclusion test of cell viability. Curr Protoc Immunol. 2015;111:A3.B.1-3. DOI: 10.1002/0471142735.ima03bs111.

18. Martín-Piedra MA, Garzón I, Oliveira AC, Alfonso-Rodríguez CA, Carriel V, Scionti G, et al. Cell viability and proliferation capability of long-term human dental pulp stem cell cultures. Cytotherapy. 2014;16(2):266-77. DOI: 10.1016/j.jcyt.2013.10.016.

19. Tirino V, Paino F, d'Aquino R, Desiderio V, De Rosa A, Papaccio G. Methods for the identification, characterization and banking of human DPSCs: current strategies and perspectives. Stem Cell Rev. 2011;7(3):608-15. DOI: 10.1007/s12015-011-9235-9.

20. Souza L, Bittar J, Silva I, Toledo O, Brígido M, Fonseca M. Comparative isolation protocols and characterization of stem cells from human primary and permanent teeth pulp. Braz J Oral Sci. 2010;9(4):427-33.

21. Perry BC, Zhou D, Wu X, Yang FC, Byers MA, Chu TM, et al. Collection, cryopreservation, and characterization of human dental pulp-derived mesenchymal stem cells for banking and clinical use. Tissue Eng Part C Methods. 2008;14(2):149-56. DOI: 10.1089/ten.tec.2008.0031.

22. Horibe H, Murakami M, Iohara K, Hayashi Y, Takeuchi N, Takei Y, et al. Isolation of a stable subpopulation of mobilized dental pulp stem cells (MDPSCs) with high proliferation, migration, and regeneration potential is independent of age. PLoS One. 2014;9(5):e98553. DOI: 10.1371/journal.pone.0098553.

23. Bressan E, Ferroni L, Gardin C, Pinton P, Stellini E, Botticelli D, et al. Donor age-related biological properties of human dental pulp stem cells change in nanostructured scaffolds. PLoSOne. 2012;7(11):e49146. DOI: 10.1371/journal.pone.0049146. 